\title{
Commentary: Epigenetic Regulation of Phosphodiesterases 2A and 3A Underlies Compromised $\beta$-Adrenergic Signaling in an iPSC Model of Dilated Cardiomyopathy
}

\author{
Lauren A. Cole, Jonathan H. Dennis and P. Bryant Chase* \\ Department of Biological Science, Florida State University, Tallahassee, FL, USA
}

Keywords: heart, dilated cardiomyopathy, troponin T, induced pluripotent stem cell, nucleus, histone methylation, epigenetic gene regulation

\section{A commentary on}

\section{OPEN ACCESS}

Edited by:

Li Zuo,

Ohio State University, USA

Reviewed by:

Han-Zhong Feng,

Wayne State University School of

Medicine, USA

Brandon Biesiadecki,

Ohio State University, USA

Jop Van Berlo,

University of Minnesota, USA

*Correspondence:

P. Bryant Chase

chase@bio.fsu.edu

Specialty section:

This article was submitted to

Striated Muscle Physiology,

a section of the journal

Frontiers in Physiology

Received: 21 July 2016 Accepted: 05 September 2016 Published: 23 September 2016

Citation:

Cole LA, Dennis JH and Chase PB (2016) Commentary: Epigenetic

Regulation of Phosphodiesterases $2 \mathrm{~A}$ and $3 A$ Underlies Compromised $\beta$-Adrenergic Signaling in an IPSC Model of Dilated Cardiomyopathy.

Front. Physiol. 7:418.

doi: 10.3389/fphys.2016.00418
Epigenetic Regulation of Phosphodiesterases 2A and 3A Underlies Compromised $\beta$-Adrenergic Signaling in an iPSC Model of Dilated Cardiomyopathy

by Wu, H., Lee, J., Vincent, L. G., Wang, Q., Gu, M., Lan, F., et al. (2015). Cell Stem Cell 17. 89-100. doi: $10.1016 /$ j.stem.2015.04.020

$\mathrm{Wu}$ et al. (2015) describe pioneering work that utilizes patient-derived induced pluripotent stem cells (iPSCs) from dilated cardiomyopathy (DCM) patients (Sun et al., 2012) and matched non-DCM relatives to study cellular mechanisms of DCM pathogenesis. They find that iPSC cardiomyocytes have proper $\beta$-adrenergic signaling while iPSCs from DCM patients exhibit impaired response to $\beta$-adrenergic agonist isoproterenol (ISO), which, physiologically, would be expected to compound the mechanical deficit associated with a mutation in troponin $\mathrm{T}$ (TnT). Surprisingly, Wu et al. (2015) find that the mechanisms of altered $\beta$-adrenergic signaling involve a direct role for TnT in epigenetic control of phosphodiesterase (PDE) expression, and that the mutation affects TnT function not only in the myofilament lattice, but also in the nucleus. This foundational work demonstrates the utility of iPSC-CMs for direct comparison of healthy vs. diseased tissues by providing a platform for identifying previously unrecognized molecular and cellular mechanisms in the progression of DCM.

The mutation studied by $\mathrm{Wu}$ et al. (2015) is a point mutation in the gene for the cardiac isoform of TnT, resulting in a single amino acid change (TNNT2 R173W) in or adjacent to TnT's tropomyosin-binding region. Many DCM mutations in myofilament proteins affect muscle function by decreasing $\mathrm{Ca}^{2+}$-sensitivity (e.g., when assaying $\mathrm{Ca}^{2+}$-dependent myofibrillar MgATPase activity, sliding speed of reconstituted thin filaments in motility assays, or force generation by permeabilized muscle preparations; Willott et al., 2010; Watkins et al., 2011); in other words, more cytoplasmic $\mathrm{Ca}^{2+}$ would be required to achieve the same functional response. This is indeed the case for the TNNT2 R173W mutation which shifts $\mathrm{Ca}^{2+}$ sensitivity of myosin S1 MgATPase activity rightward (toward higher $\left[\mathrm{Ca}^{2+}\right]$ ) by almost $0.1 \mathrm{pCa}$ units, with little or no effect on the maximum MgATPase activity or the maximum sliding speed of thin filaments in motility assays (Sommese et al., 2013). This altered $\mathrm{Ca}^{2+}$-responsiveness of the myofilaments almost certainly results directly in reduced mechanical function of the heart during systole, to the detriment of the DCM patient. Remodeling of the DCM heart, however, depends in part on changes in gene expression. Mechanisms of altered gene regulation in cardiomyopathies have typically 
focused on changes in $\mathrm{Ca}^{2+}$-signaling, mechanosensing, and/or energy metabolism (Frey et al., 2004; Ahmad et al., 2005; Kataoka et al., 2007; Lakdawala et al., 2012; Moore et al., 2012; LeWinter and Granzier, 2014). Wu et al. (2015) invoke a novel and more direct role of $\mathrm{TnT}$ in gene regulation.

Wu et al. (2015) found that TnT was present in one-third of nuclei from iPSCs derived from DCM patients with the TNNT2 R173W mutation, compared to $\sim 5 \%$ of nuclei of iPSCs derived from normal individuals. TnT is an abundant myofilament protein present in the sarcomere, responsible for attachment of the troponin complex to tropomyosin and transmission of the $\mathrm{Ca}^{2+}$ signal that activates systolic cardiac contraction (Figure 1). Although $\mathrm{TnT}$ contains a strong nuclear localization signal (NLS), its functional role in the nucleus of striated muscle myocytes is poorly understood (Bergmann et al., 2009; Zhang et al., 2015, 2016). Identification of TnT interacting proteins in the nucleus is critical to understanding its function.

Wu et al. (2015) performed co-immunoprecipitation studies in cardiomyocyte nuclear extracts to identify TnT interacting proteins. They found that $\mathrm{TnT}$ is associated with histone demethylases KDM1A and KDM5A, as well as histone H3. Furthermore, they characterized chromatin patterns of the PDE $2 \mathrm{~A}$ and $3 \mathrm{~A}$ genes, where the authors found significant increases of activation marks (H3K4me3) and decreased repressive marks (H3K27me3) in sequences defined by the authors as regions 1 and 2. Assuming high specificity for the various antibodies used throughout their assays, these results suggest that $\operatorname{TnT}$ normally plays a role in the epigenetic

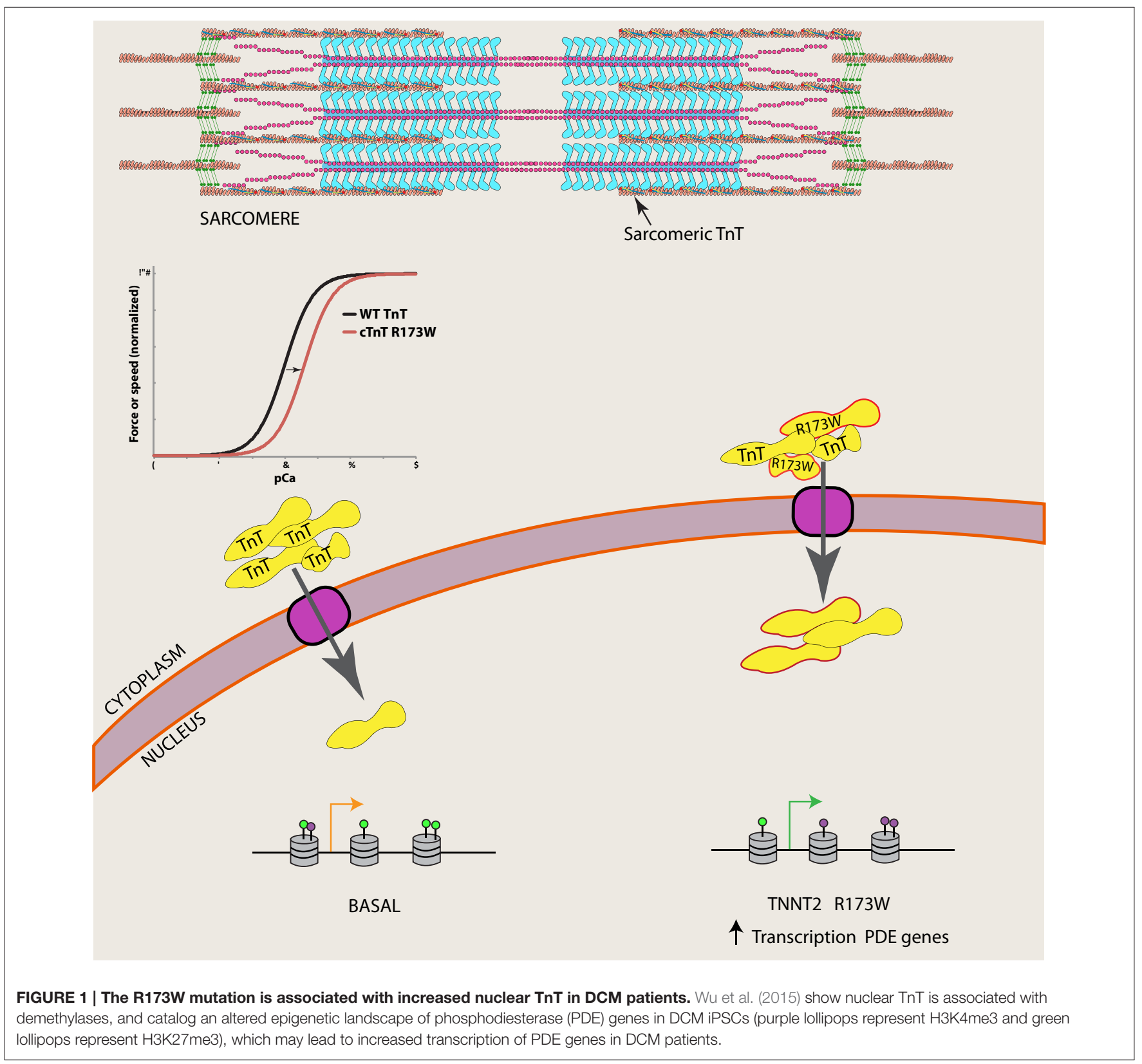


regulation of at least these PDE genes. Their study furthermore demonstrates that a TnT mutation not only affects sarcomeric function, but also contributes to the improper regulation of both nuclear localization of TnT and PDE gene expression in DCM patients (Figure 1). Precise epigenetic regulation of cardiomyocyte differentiation as well as regulation of expression in a cell-type-specific manner has been recently documented, demonstrating this layer of information is critical for understanding cardiomyocyte (dys)function (Paige et al., 2012; Wamstad et al., 2012; O'Meara and Lee, 2015; Preissl et al., 2015). An improper epigenetic landscape likely contributes to inappropriate regulation of many genes, and it will be important for future work to explore other known DCM mutations in the context of genome architecture.

Wu et al. (2015) demonstrate the use of iPSCs to study a prevalent heart disease and determine a novel role of epigenetic regulation in pathogenesis of DCM. This finding demonstrates that mutations in mechanical proteins that lead to DCM pathogenesis via sarcomere dysfunction can also be exacerbated by regulation of epigenomic state. Nuclear localization of cardiac troponin I (TnI), cardiac troponin C (TnC), and cardiac TnT has been shown in rat neonatal ventricular cardiomyocytes, but their relationship with one another, and presumably tropomyosin and actin, in the nucleus has yet to be clearly established (Asumda and

\section{REFERENCES}

Ahmad, F., Seidman, J. G., and Seidman, C. E. (2005). The genetic basis for cardiac remodeling. Annu. Rev. Genomics Hum. Genet. 6, 185-216. doi: 10.1146/annurev.genom.6.080604.162132

Asumda, F. Z., and Chase, P. B. (2012). Nuclear cardiac troponin and tropomyosin are expressed early in cardiac differentiation of rat mesenchymal stem cells. Differentiation 83, 106-115. doi: 10.1016/j.diff.2011.10.002

Bergmann, O., Bhardwaj, R. D., Bernard, S., Zdunek, S., Barnabé-Heider, F., Walsh, S., et al. (2009). Evidence for cardiomyocyte renewal in humans. Science 324, 98-102. doi: 10.1126/science.1164680

Brunet, N. M., Chase, P. B., Mihajlović, G., and Schoffstall, B. (2014). Ca( $\left.{ }^{2+}\right)-$ regulatory function of the inhibitory peptide region of cardiac troponin $\mathrm{I}$ is aided by the $\mathrm{C}$-terminus of cardiac troponin $\mathrm{T}$ : effects of familial hypertrophic cardiomyopathy mutations cTnI R145G and cTnT R278C, alone and in combination, on filament sliding. Arch. Biochem. Biophys. 552-553, 11-20. doi: 10.1016/j.abb.2013.12.021

Chase, P. B., Szczypinski, M. P., and Soto, E. P. (2013). Nuclear tropomyosin and troponin in striated muscle: new roles in a new locale? J. Muscle Res. Cell Motil. 34, 275-284. doi: 10.1007/s10974-013-9356-7

Frey, N., Barrientos, T., Shelton, J. M., Frank, D., Rütten, H., Gehring, D., et al. (2004). Mice lacking calsarcin-1 are sensitized to calcineurin signaling and show accelerated cardiomyopathy in response to pathological biomechanical stress. Nat. Med. 10, 1336-1343. doi: 10.1038/nm1132

Hershberger, R. E., Hedges, D. J., and Morales, A. (2013). Dilated cardiomyopathy: the complexity of a diverse genetic architecture. Nat. Rev. Cardiol. 10, 531-547. doi: 10.1038/nrcardio.2013.105

Ho, C. Y., Charron, P., Richard, P., Girolami, F., Van Spaendonck-Zwarts, K. Y., and Pinto, Y. (2015). Genetic advances in sarcomeric cardiomyopathies: state of the art. Cardiovasc. Res. 105, 397-408. doi: 10.1093/cvr/c vv025

Kataoka, A., Hemmer, C., and Chase, P. B. (2007). Computational simulation of hypertrophic cardiomyopathy mutations in troponin I: influence of increased myofilament calcium sensitivity on isometric force, ATPase and $\left[\mathrm{Ca}^{2+}\right]$ i. J. Biomech. 40, 2044-2052. doi: 10.1016/j.jbiomech.2006.09.026

Lakdawala, N. K., Thune, J. J., Colan, S. D., Cirino, A. L., Farrohi, F., Rivero, J., et al. (2012). Subtle abnormalities in contractile function are an early manifestation
Chase, 2012). Interestingly, co-IP data from Wu et al. (2015) did not identify TnI or tropomyosin as interacting partners of nuclear TnT. It may be the case that these partners in thin filament regulation have independent roles in the nucleus. Because these proteins are often mutated in DCM patients, further studies are necessary to not only delineate the function of these proteins in the nucleus in normal individuals, but to determine whether the unique mechanisms identified by $\mathrm{Wu}$ et al. (2015) (i.e., unexpected changes in nuclear localization and unexpected interactions with other molecules, which in this instance affect epigenetic regulation of physiologically important genes) are specific only to the R173W mutation in TNNT2, or if they are more commonly associated with other myofilament protein mutations and other mutations that cause cardiomyopathies (Schoffstall et al., 2011; Chase et al., 2013; Hershberger et al., 2013; Teekakirikul et al., 2013; Brunet et al., 2014; Ho et al., 2015; Teo et al., 2015). The involvement of nuclear mechanical proteins in regulation of chromatin, and thus expression, is a new and important aspect of DCM pathogenesis.

\section{AUTHOR CONTRIBUTIONS}

LC and PC wrote and edited the commentary, JD edited the commentary.

of sarcomere mutations in dilated cardiomyopathy. Circ. Cardiovasc. Genet. 5, 503-510. doi: 10.1161/CIRCGENETICS.112.962761

LeWinter, M. M., and Granzier, H. L. (2014). Cardiac titin and heart disease. J. Cardiovasc. Pharmacol. 63, 207-212. doi: 10.1097/FJC.0000000000000007

Moore, J. R., Leinwand, L., and Warshaw, D. M. (2012). Understanding cardiomyopathy phenotypes based on the functional impact of mutations in the myosin motor. Circ. Res. 111, 375-385. doi: 10.1161/CIRCRESAHA.110.223842

O'Meara, C. C., and Lee, R. T. (2015). Peering into the cardiomyocyte nuclear epigenetic state. Circ. Res. 117, 392-394. doi: 10.1161/CIRCRESAHA.115.307093

Paige, S. L., Thomas, S., Stoick-Cooper, C. L., Wang, H., Maves, L., Sandstrom, R., et al. (2012). A temporal chromatin signature in human embryonic stem cells identifies regulators of cardiac development. Cell 151, 221-232. doi: 10.1016/j.cell.2012.08.027

Preissl, S., Schwaderer, M., Raulf, A., Hesse, M., Grüning, B. A., Köbele, C., et al. (2015). Deciphering the epigenetic code of cardiac myocyte transcription. Circ. Res. 117, 413-423. doi: 10.1161/CIRCRESAHA.115.306337

Schoffstall, B., LaBarbera, V. A., Brunet, N. M., Gavino, B. J., Herring, L., Heshmati, S., et al. (2011). Interaction between troponin and myosin enhances contractile activity of myosin in cardiac muscle. DNA Cell Biol. 30, 653-659. doi: $10.1089 /$ dna.2010.1163

Sommese, R. F., Nag, S., Sutton, S., Miller, S. M., Spudich, J. A., and Ruppel, K. M. (2013). Effects of troponin T cardiomyopathy mutations on the calcium sensitivity of the regulated thin filament and the actomyosin crossbridge kinetics of human $\beta$-cardiac myosin. PLoS ONE 8:e83403. doi: 10.1371/journal.pone.0083403

Sun, N., Yazawa, M., Liu, J., Han, L., Sanchez-Freire, V., Abilez, O. J., et al. (2012). Patient-specific induced pluripotent stem cells as a model for familial dilated cardiomyopathy. Sci. Transl. Med. 4:130ra47. doi: 10.1126/scitranslmed.3003552

Teekakirikul, P., Kelly, M. A., Rehm, H. L., Lakdawala, N. K., and Funke, B. H. (2013). Inherited cardiomyopathies: molecular genetics and clinical genetic testing in the postgenomic era. J. Mol. Diagn. 15, 158-170. doi: 10.1016/j.jmoldx.2012.09.002

Teo, L. Y., Moran, R. T., and Tang, W. H. (2015). Evolving approaches to genetic evaluation of specific cardiomyopathies. Curr Heart Fail Rep. 12, 339-349. doi: 10.1007/s11897-015-0271-7 
Wamstad, J. A., Alexander, J. M., Truty, R. M., Shrikumar, A., Li, F., Eilertson, K. E., et al. (2012). Dynamic and coordinated epigenetic regulation of developmental transitions in the cardiac lineage. Cell 151, 206-220. doi: 10.1016/j.cell.2012.07.035

Watkins, H., Ashrafian, H., and Redwood, C. (2011). Inherited cardiomyopathies. N. Engl. J. Med. 364, 1643-1656. doi: 10.1056/NEJMra090 2923

Willott, R. H., Gomes, A. V., Chang, A. N., Parvatiyar, M. S., Pinto, J. R., and Potter, J. D. (2010). Mutations in Troponin that cause HCM, DCM AND RCM: what can we learn about thin filament function? J. Mol. Cell. Cardiol. 48, 882-892. doi: 10.1016/j.yjmcc.2009.10.031

Wu, H., Lee, J., Vincent, L. G., Wang, Q., Gu, M., Lan, F., et al. (2015). Epigenetic regulation of phosphodiesterases $2 \mathrm{~A}$ and $3 \mathrm{~A}$ underlies compromised $\beta$-adrenergic signaling in an iPSC model of dilated cardiomyopathy. Cell Stem Cell 17, 89-100. doi: 10.1016/j.stem.2015.04.020

Zhang, T, Pereyra, A. S., Wang, Z. M., Birbrair, A., Reisz, J. A., Files, D. C., et al. (2016). Calpain inhibition rescues troponin T3 fragmentation, increases Cav1.1, and enhances skeletal muscle force in aging sedentary mice. Aging Cell 15, 488-498. doi: 10.1111/acel.12453
Zhang, T., Taylor, J., Jiang, Y., Pereyra, A. S., Messi, M. L., Wang, Z. M., et al. (2015). Troponin T3 regulates nuclear localization of the calcium channel Cav $\beta 1$ a subunit in skeletal muscle. Exp. Cell Res. 336, 276-286. doi: 10.1016/j.yexcr.2015.05.005

Conflict of Interest Statement: The authors declare that the commentary was written in the absence of any commercial or financial relationships that could be construed as a potential conflict of interest.

The reviewer $\mathrm{BB}$ and handling Editor declared their shared affiliation, and the handling Editor states that the process nevertheless met the standards of a fair and objective review.

Copyright $\odot 2016$ Cole, Dennis and Chase. This is an open-access article distributed under the terms of the Creative Commons Attribution License (CC BY). The use, distribution or reproduction in other forums is permitted, provided the original author(s) or licensor are credited and that the original publication in this journal is cited, in accordance with accepted academic practice. No use, distribution or reproduction is permitted which does not comply with these terms. 